\title{
Steal syndrome following an arteriovenous fistula - case report and treatment option
}

\author{
Cristian Ene Roată ${ }^{1}$, Corneliu Moroșanu ${ }^{1}$, Silviu Tiberiu Makkai-Popa ${ }^{* 1}$, Adrian \\ Constantin Covic ${ }^{2}$
}

${ }^{1}$ Surgery Department, "Grigore T. Popa" University of Medicine and Pharmacy, Iași, ${ }^{2}$ Department of Internal Medicine - Nephrology, "Grigore T. Popa" University of Medicine and Pharmacy lași, Romania

\begin{abstract}
The paper presents the case of a 48 year-old diabetic patient that underwent hemodialysis for a long period of time and presented to our hospital with digital hypoperfusion symptoms following an arteriovenous fistula between the humeral artery and the basilic vein. The anamnesis of the patient reveals prior circulatory problems and in the case of a previous fistula the patient required the amputation of the distal phalanx of the left index finger. The arterial theft syndrome was dealt with in this case by using a simple method based on techniques of flow diminution through the vein, assessing the calibration by means of clinical criteria such as capillary flow, presence of thrill and decrease in pain levels. A 1 year follow-up showed the fistula was still viable and the symptoms diminished to a level that no longer interfered with the lifestyle of the patient.
\end{abstract}

Keywords: vascular access steal syndrome, preserve vascular access, hypoperfusion ischemic syndrome, arteriovenous fistulas

\section{Introduction}

Arteriovenous anastomosis causes a large amount of the blood flow to be shunted into the superficial venous system because the venous peripheral resistance is significantly lower than the one in the arterial system. One compensatory mechanism to this phenomenon is the arterial relaxation which helps by increasing the blood flow not only through the anastomosis but also distal to it. One other compensatory phenomenon is neoangiogenesis as a response to the decrease in blood flow distal to the anastomosis [1]. However, all of these

Received: July 2015; Accepted after review: August 2015; Published: September 2015.

${ }^{*}$ Corresponding author: Assist. Prof. Silviu Tiberiu Makkai-Popa, MD, PhD, Surgery Department, "Grigore T. Popa" University of Medicine and Pharmacy lasi, 16, Universitatii Street, 700115, lasi, Romania

E-mail:mpsilviu@yahoo.com compensatory mechanisms are insufficient in diabetic patients who have vascular problems such as accelerated atherosclerosis and microangiopathy which make relaxation impossible.

The digital hypoperfusion syndrome is a result of the arterial theft and can evolve progressively over a long period of time with paresthesia, pain and pale discoloration of the skin when exposed to cold. But in some high risk patients they can also present as an acute syndrome even before the maturation of the fistula with intense pain, muscle rigidity, pale and cold extremity, paresis and different degrees of impairment. If the symptoms are left untreated they are followed by necrosis.

\section{Case report}

We herein describe the case of a 48 yearold patient diagnosed with insulin-dependent 
diabetes mellitus since the age of 21 . He has been on hemodialysis for 10 years and has presented major vascular complications during this time. He initially had several fistulas in his right upper arm which constantly complicated with ischemic symptoms such as paresthesia and pain. Three years before the current presentation he underwent his first arteriovenous fistula in his left arm that was not functional which meant the patient had to undergo surgery for a second fistula to be created between the humeral artery and the left cephalic vein.

Post-surgery he developed necrotic lesions of the fingers of his left hand that required an amputation of the first phalanx of his left index finger. Shortly after he presented a thrombosis of the fistula and a "long life" catheter was inserted in the right internal jugular vein that was used for 2 and a half years (30 months), before the patient was admitted in our department.
At his first presentation in our department we noted the lack of pulse in both radial arteries, an exhausted venous capital in the right upper arm and the possibility of performing an arteriovenous fistula between the humeral artery and the medio-basilic vein in the left arm. Such an anastomosis was performed in a side-to-end fashion. Postoperatively we noted the left upper extremity was colder than the right one and at the $12^{\text {th }}$ postoperative day the patient developed paresthesia and then increasing pain starting with the $15^{\text {th }}$ postoperative day and finally muscular rigidity and functional impairment. At 6 weeks follow-up consultation, when the patient returned for the surfacing of the basilic vein he had permanent spontaneous pain in his fingers, muscular rigidity and 2 small necrotic lesions on the anterior aspect of the $2^{\text {nd }}$ phalanx of the $5^{\text {th }}$ finger and at its base on the posterior aspect of the hand (Figure 1).

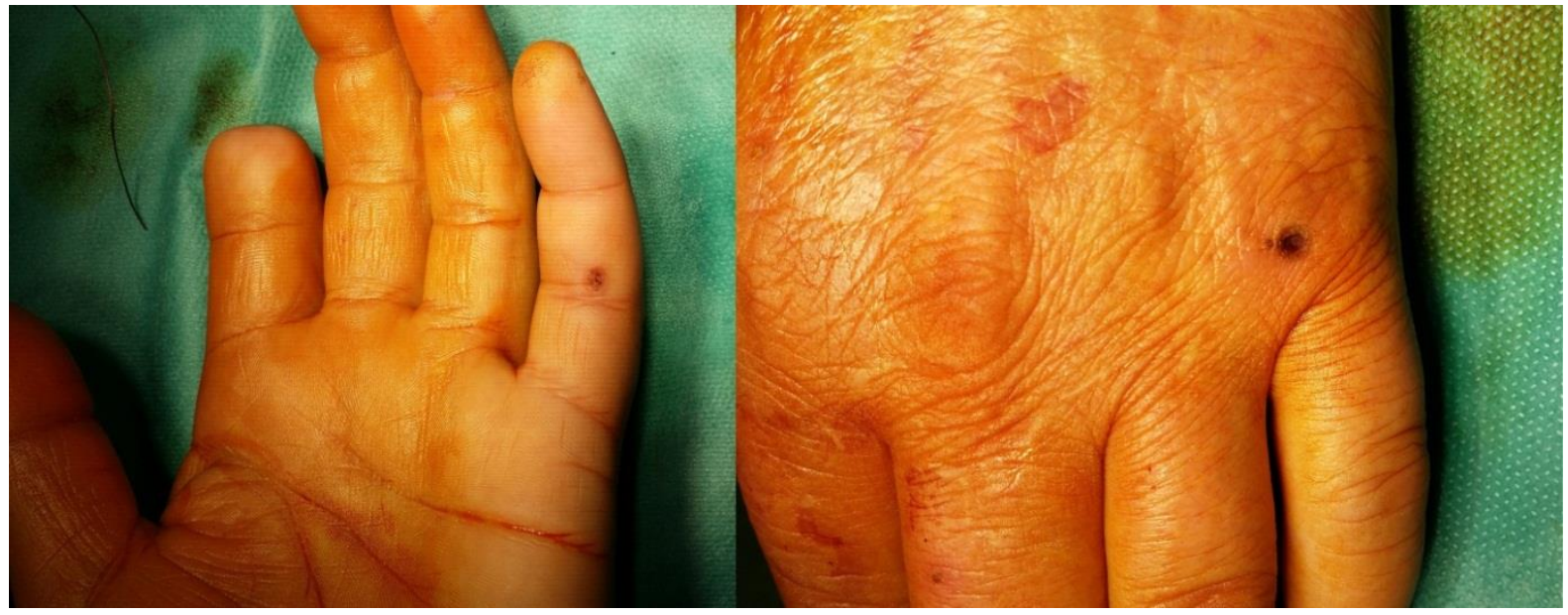

Fig. 1. Necrotic lesions following the arteriovenous anastomosis

The surgical procedure was aimed at surfacing the basilic vein. The patient was told to follow his regular treatment and to lead a normal life-style in the week before the surgical procedure so that the values of the blood pressure could be within the normal range. Preoperatively we used ultrasound to mark the skin projection of the basilic vein. The patient was placed in supine position with his left arm in external rotation and abduction at a 90 degrees angle. Under local anesthesia with $1 \%$ Xylocaine an incision was made on the previously marked line and the vein was dissected and isolated circumferentially over a length of about $20 \mathrm{~cm}$. All the collateral vessels were sectioned and ligated (Figure 2). We waited for 20 minutes to check if the patient felt any major changes in his symptoms and the patient reported no significant changes, only a mild decrease in pain levels.

We then performed a stenosing ligature using a surgical thread (0 silk) passed twice circumferentially around the vein at about 1.5- 
$2 \mathrm{~cm}$ from the anastomosis. The suture was then tied progressively with only one loop of the knot until we obtained capillary pulse distal to the anastomosis. We calibrated the stenosis we induced by constantly checking for the capillary pulse as a means to assess the flow distal to the anastomosis and by checking the vein and making sure the vein distal to the ligature is kept dilated as a means to check for a good flow through the vein (Figure 3).

Thirty minutes after the procedure the patient regained mobility in his fingers and the pain on palpation of the fingers disappeared. We then performed 2 more knots and the fascia and subcutaneous fat were closed. The

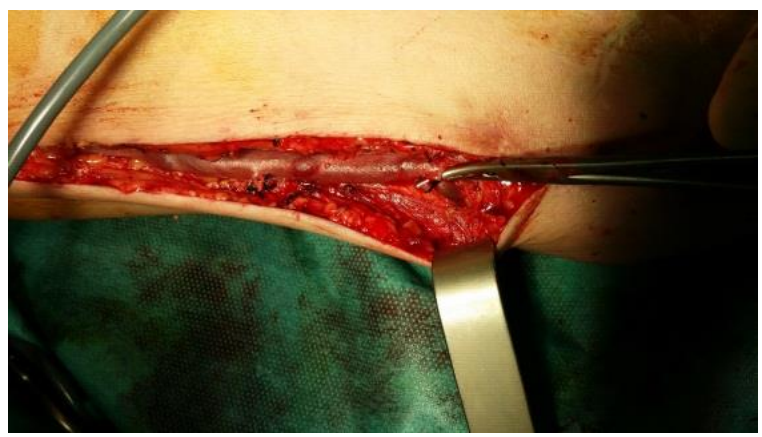

Fig. 2. Ligature of venous collaterals

\section{Discussions}

Arterial theft after arteriovenous fistulas is a frequent phenomenon in diabetic patients due to the vascular complications of diabetes which limit the ability of the arteries to relax in order to reduce peripheral resistance and increase blood flow distal to the anastomosis. Current data from the literature reports a percentage of $3.7-5.0 \%$ of diabetic patients that undergo dialysis, with clinically manifest digital hypo-perfusion after arteriovenous fistulas [2].

Lazarides et al. calculated a systolic pressure index (SPI - ratio between the postoperative systolic pressure in the forearm bearing the fistula and the systolic pressure at the same level on the controlateral forearm) in 69 patients and concluded that in $94 \%$ of these patients the SPI is beneath 0.8 , which means a clinically assessable perfusion deficit. However, only $8 \%$ of these patients developed a symptomatic hypoperfusion syndrome [3]. vein was placed subcutaneously and the skin was sutured using separate suture points. After 14 days the skin sutures were removed and after 21 days the patient started hemodialysis.

A follow-up was performed at 1, 3 and 12 postoperative months after the stenosing ligature of the vein. At 12 months the fistula was still functional and the only symptoms were pain under effort conditions and the fact that the left hand was colder than the right hand. Hand mobility was fully recovered and the remaining symptoms were not interfering with the patient's life style.

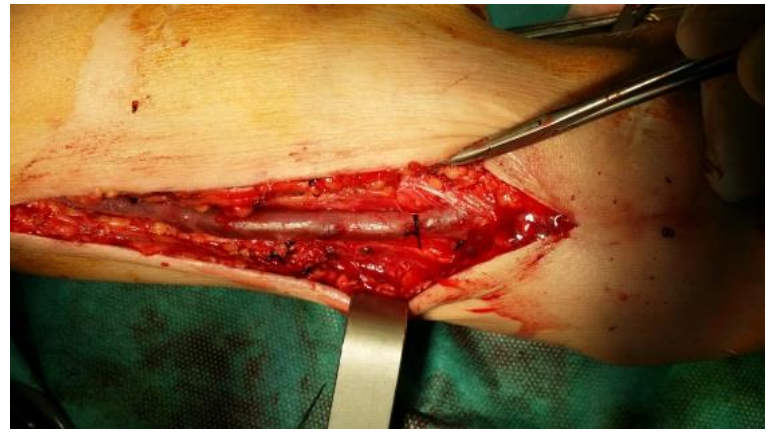

Fig. 3. Final aspect of the stenosing ligature

Our case is a particular situation in which in a young patient it is impossible to obtain native or prosthetic fistula without endangering the patient's limb because vascular damage induced by a long evolving diabetes. In 2010, Majid Moini et al. reported the results of a prospective study on 50 patients in which they performed the ligation of venous perforating vessels right after the arteriovenous anastomosis. The results of his study show that such a technique is successful in preventing the digital hypoperfusion syndrome and venous hypertension despite the fact that in his study $22 \%$ of the patients were diabetic patients [4]. In our view the ligation of the collaterals is extremely important because it decreases the amount of blood deviated towards the venous system, thus enhancing the distal perfusion.

Some of the best solutions presented in the literature from a hemodynamic point of view are the proximalization of the arterial inflow [5] and the Distal Revascularization and 
Interval Ligation (DRIL) [6]. However, patients with digital hypoperfusion present multiple comorbidities (dialysis, insulin-dependent diabetes) and these techniques require a prosthetic material and general anesthesia, both increasing the risk of postoperative or intraoperative complications in these patients. In our view such methods should be used as a last resort because of the anesthetic and septic risks involved especially after thrombosis of the fistula.

Our method can be applied to patients in whom the perfusion distal to the elbow, before the fistula, allows for a decrease in blood flow without ischemic symptoms. In time, due to angiogenesis and collateral circulation development, the perfusion ameliorates. However, in cases with critical ischemia preexisting the fistula our method does not yield results and the solution is in our opinion the proximalization of the arterial inflow or the DRIL technique.

Another technique of increasing the blood flow through the fistula in order to increase perfusion suggests the revision using distal inflow (RUDI) from one of the arteries distal to the fistula. In our opinion such a technique has the same problems as the critical ischemia situations and the literature reports regarding this technique are scarce $[7,8]$.

Techniques similar to ours are the vein plication technique [9] and the banding technique [10]. The vein plication is a procedure that once finished does not allow any readjustment and thus the risk of losing the fistula and of having performed an ineffective surgical gesture is high. The banding however, allows for adjustments but the calibration is much more complicated and requires a long learning curve in our opinion.

The MILLER technique was originally described in 2006 and consists in performing a stenosing ligature and calibrating the stenosis

\section{References}

1. Tynan-Cuisinier GS, Berman SS. Strategies for predicting and treating access induced ischemic steal syndrome. Eur J Vasc Endovasc Surg 2006; 32(3):309-315.

2. Tordoir JH, Dammers R, Van der Sande FM. by means of a $4-5 \mathrm{~mm}$ balloon placed inside the vein, which means a fixed diameter of the vein for all patients [11]. The method we use, by comparison, allows for a much more flexible calibration, according to each patient's hemodynamic conditions. The only requirements of our technique being to maintain a balance between the blood flow through the stenosis and the flow through the vein distal to the ligature. In order to assess whether or not the blood flow is sufficient we use the assessment of the capillary pulse or the remission of the symptoms.

\section{Conclusions}

The method we propose can be used as a first line method to deal with arterial theft secondary to an arteriovenous fistula in dialysis patients because it is easy to perform and it requires a minimal aggression on the patient.

In cases where our method fails, it is our opinion that the methods that increase the blood flow to the fistula, such as the arterial inflow proximalization technique and the DRIL technique must be used to save the fistula and ensure perfusion distal to the fistula.

The important aspect in techniques that reduce the venous blood flow (our technique, the banding, vein plication and MILLER techniques) is finding the balance between appropriate distal perfusion and the blood flow through the vein. In this context our method can be a very good option because it allows us to calibrate the stenosis depending on the hemodynamic condition of each patient. More than that our method is easy to use because all signs are clinical signs and do not require any supplementary tests or maneuvers during the procedure.
Upper extremity ischemia and hemodialysis vascular access. Eur $J$ Vasc Endovasc Surg 2004; 27:1-5.

3. Lazarides MK, Staamos DN, Panagopoulos GN, Tzilalis VD, Eleftheriou GJ, Dayantas JN. 
Indications for surgical treatment of angioaccess-induced arterial 'steal'. J Am Coll Surg 1998; 187(4):422-426.

4. Moini M, Rasouli MR, Nouri M. Ligation of the perforating vein: a treatment for steal syndrome in side-to-side elbow arteriovenous fistula. Ann Vasc Surg 2008; 22(2):307.

5. Zanow J, Kruger U, Scholz H. Proximalization of the arterial inflow: a new technique to treat access-related ischemia. J Vasc Surg 2006; 43:1216-1221.

6. Walz P, Ladowski JS, Hines A. Distal Revascularization and Interval Ligation (DRIL) Procedure for the Treatment of Ischemic Steal Syndrome after Arm Arteriovenous Fistula. Ann Vasc Surg 2007; 21:468-473.

7. Minion David J, Moore E, Endean E. Revision Using Distal Inflow: A Novel Approach to
Dialysis-associated Steal Syndrome. Ann Vasc Surg 2005; 19(5):625-628.

8. Gupta N, Yuo $\mathrm{TH}$, Konig G $4^{\text {th }}$, et al. Treatment strategies of arterial steal after arteriovenous access. J Vasc Surg 2011; 54:162-167.

9. Yaghoubian A, de Virgilio C. Plication as Primary Treatment of Steal Syndrome in Arteriovenous Fistulas. Ann Vasc Surg 2009; 23:103-107.

10. Schneider CG, Gawad K, Strate $T$, et al. Tbanding: A technique for flow reduction of a hyperfunctioning arteriovenous fistula. J Vasc Surg 2006; 43:402-405.

11. Goel N, Miller GA, Jotwani MC, Licht J, Schur I, Arnold WP. Minimally invasive limited ligation endoluminal- assisted revision (MILLER) for treatment of dialysis access-associated steal syndrome. Kidney Int 2006; 70(4):765-770. 